\title{
Actitud de los profesores ante el uso de las TIC en la Facultad de Ciencias Médicas de Matanzas
}

\author{
Attitude of teachers towards the use of ICT at the faculty of Medical Sciences \\ of Matanzas \\ Lorayne Almanza Santana ${ }^{1}$, Sandra Naranjo Rodríguez. ${ }^{2}$, Lissett Rodríguez Almanza. ${ }^{3} \&$ \\ Rachel Delgado Almanza. ${ }^{4}$
}

Recibido: 07-09-2020 / Revisado: 31-10-2020 /Aceptado: 21-11-2020/ Publicado: 02-01-2021

DOI: https://doi.org/10.33262/anatomiadigital.v4i1.1479

Abstract.

Introduction: The continuous improvement of the Cuban educational system, which has taken place in recent years, conditions the need to renew, within the framework of Higher Education Centers, the study plans from a perspective that responds to the demands associated with the process of Universalization of Education that takes place in them, which implies that the

\section{Resumen.}

Introducción: El perfeccionamiento continuo del sistema educativo cubano, que tiene lugar en los últimos años, condiciona la necesidad de renovar, en el marco de los Centros de Educación Superior, los planes de estudios desde una perspectiva que responda a las exigencias asociadas al proceso de Universalización de la Educación que en ellos tiene lugar,

\footnotetext{
${ }^{1}$ Universidad de Ciencias Médicas de Matanzas, Facultad de Ciencias Médicas “Juan Guiteras Gener”, Matanzas. Cuba. lorayne.mtz@infomed.sld.cu (i) https://orcid.org/0000-0001-9758-3617

${ }^{2}$ Universidad de Ciencias Médicas de Matanzas, Facultad de Ciencias Médicas “Juan Guiteras Gener”, Matanzas. Cuba. sandranaranjo.mtz@infomed.sld.cu iD https://orcid.org/0000-0001-5035-3101

${ }^{3}$ Universidad de Ciencias Médicas de Matanzas, Facultad de Ciencias Médicas “Juan Guiteras Gener”, Matanzas. Cuba. lisset.mtz@infomed.sld.cu iD https://orcid.org/0000-0001-6899-2489

${ }^{4}$ Universidad de Ciencias Médicas de Matanzas, Facultad de Ciencias Médicas “Juan Guiteras Gener”, Matanzas, Cuba. rachel.est@infomed.sld.cu (D https://orcid.org/0000-0002-1320-9450
} 
university as a social institution directs its work to achieve prominent results; in the introduction, innovation and creation of technologies Objective: To assess the attitude of teachers towards the use of ICT in the Faculty of Medical Sciences of Matanzas. Methods: A quantitative study was carried out in the Faculty of Medical Sciences of Matanzas in the period of January-May 2019, to evaluate the attitude of the professors, before the use that the professors of the Faculty of Sciences make of ICT Matanzas Doctors. Results: The results showed that the professors of the Faculty of Medical Sciences of Matanzas have an excellent attitude towards the use of information and communication technologies in undergraduate teaching Conclusions: Undoubtedly, new technologies can provide means to improve teaching-learning processes, but their use in favor or against a more developed society will depend largely on the attitude of teachers as the main guides of the process.

Keywords: ICT, teaching-learning process, University lo cual implica que la universidad como institución social dirija su trabajo a alcanzar resultados prominentes; en la introducción, innovación y creación de tecnologías Objetivo: Valorar la actitud de los profesores ante el uso de la TIC en la Facultad de Ciencias Médicas de Matanzas. Metodología: Se realizó un estudio cuantitativo en la Facultad de Ciencias Médicas de Matanzas en el período de enero -mayo del 2019, para evaluar la actitud de los profesores, ante el uso que realizan de las TIC los profesores de la Facultad de Ciencias Médicas de Matanzas. Resultados: Los resultados mostraron que los profesores de la Facultad de Ciencias Médicas de Matanzas poseen una actitud excelente ante el uso de las tecnologías de la información y las comunicaciones en la docencia de pregrado Conclusiones: Sin duda las nuevas tecnologías pueden suministrar medios para la mejora de los procesos de enseñanza aprendizaje pero su utilización a favor o en contra de una sociedad más desarrollada, dependerá en gran medida de la actitud, de los profesores como guías principales del proceso

Palabras claves: TIC, proceso enseñanza-aprendizaje, Universidad

\section{Introducción.}

El perfeccionamiento continuo del sistema educativo cubano, que tiene lugar en los últimos años, condiciona la necesidad de renovar, en el marco de los Centros de Educación Superior, los planes de estudios desde una perspectiva que responda a las exigencias 
asociadas al proceso de Universalización de la Educación que en ellos tiene lugar, lo cual implica que la universidad como institución social dirija su trabajo a alcanzar resultados prominentes; en la introducción, innovación y creación de tecnologías

El uso de las tecnologías de la información y las comunicaciones dentro del sector salud es algo que llegó en respuesta a la necesidad de cambio que tienen que asumir los educandos y los educadores, en el desarrollo del proceso enseñanza aprendizaje para lograr un profesional capaz de autogestionar y autoevaluar su conocimiento con el fin de responder, con un pensamiento lógico y creador, a los intereses de Cuba y del Mundo.

Para responder a las necesidades de la sociedad la universidad debe ofrecer una respuesta educacional pertinente con el propósito fundamental de educar, formar, investigar, promover, generar y difundir conocimientos, así como desarrollar mecanismos de cooperación e integración. (Vela, 2015)

El uso de las tecnologías de la información y las comunicaciones transforma la obtención, procesamiento, socialización y conservación de la información, así como el modo de comunicación entre los sujetos. Las mismas vienen a ocupar un lugar preponderante en los actuales procesos formativos universitarios, lo cual se plantea desde diferentes perspectivas por autores como Rivero (2014) y Vialart (2015).

Junto a ellas transita el profesor como actor principal en la guía y conducción de un modelo pedagógico que realmente logre motivar e incluir al joven universitario que hoy se tiene sentado en las aulas y que se parece más a su época que al referente educativo con que se formaron esos docentes (Rodriguez, 2017).

La informatización tiene una importancia fundamental y es imprescindible en los tiempos que corren, pero ello no es posible sin que se genere un cambio en la actitud de los profesionales y se aumente el aprovechamiento de las mismas en función de elevar el proceso docente, contribuyendo a la mejor preparación de los educandos y a la superación de los educadores. (Rodero, 2015)

Por lo que los autores de esta investigación tienen como aspiración contribuir a la preparación de los profesores para fortalecer el uso de las tecnologías en función del proceso de enseñanza - aprendizaje en el pregrado, las insuficiencias que se encontradas revelan, que son escasas las investigaciones que valoran el nivel de conocimiento y uso que se hace de las tecnologías los profesores de pregrado en la facultad de Ciencias Médicas de Matanzas. Para dar respuesta al problema planteado, los autores se proponen el cumplimiento del siguiente objetivo: Valorarla actitud de los profesores ante el uso de la TIC en la Facultad de Ciencias Médicas de Matanzas. 


\section{Metodologia.}

Se realizó un estudio cuantitativo en la Facultad de Ciencias Médicas de Matanzas en el período de enero - mayo del 2019, para evaluar la actitud de los profesores ante el uso de la TIC en la Facultad de Ciencias Médicas de Matanzas.

El universo de estudio estuvo constituido por los 74 profesores pertenecientes al claustro de área básica (1ro y 2do año) de la carrera de medicina.

Se aplicó un cuestionario tipo escala Likert, compuestos por 31 ítems, que ofrecen la opción de responder empleando cinco categorías: nunca (1), a veces (2), frecuentemente (3), casi siempre (4) y siempre (5). Además, el diseño del cuestionario concibió tres dimensiones: a) Actitud ante las TIC, b) Nivel de conocimiento sobre las TIC y c) Uso que se realiza de las TIC.

Se trabajó con las variables sexo con la categoría femenina y masculina, edad donde se utilizaron las categorías siguientes: de 20 a 39, de 40 a 59 y de 60 a 80 . la variable actitud donde las categorías utilizadas fueron: Mala: menos de 69.9, aceptable: entre 70 y 89.9 y excelente: entre 90 y 100.

Esta investigación respetó los postulados de la ética de la investigación. Tuvo como objetivo esencial el puramente científico, sin afectaciones del medio ambiente, ni riesgos predecibles. Para realizar la encuesta, se le informó al entrevistado de los objetivos perseguidos con la investigación y se le solicitó su consentimiento. La información obtenida no se empleó para otros fines fuera del marco de la investigación. Los datos primarios se manejaron con discreción para la investigación.

\section{Resultados}

\section{Resultados cuantitativos de la aplicación del instrumento evaluativo}

En la tabla 1 se realizó la distribución general teniendo en cuenta el sexo de los profesores que laboran en el área de ciencias básica de la carrera de Medicina en la Facultad de Ciencias Médicas de Matanzas. La mayor cantidad pertenecen al sexo femenino con un $82.4 \%$ y el resto son masculinos con $17.6 \%$.

Tabla 1. Distribución de los docentes atendiendo al sexo y edad

\begin{tabular}{lll}
\multirow{2}{*}{ Sexo } & \multicolumn{2}{c}{ Cantidad } \\
\cline { 2 - 3 } & No. & $\%$ \\
\hline Femenino & 61 & 82.4 \\
Masculino & 13 & 17.6 \\
Total & 74 & 100 \\
\hline
\end{tabular}

Fuente: Elaboración propia 
En la tabla 2 se realizó la distribución por grupos de edades de los profesores que hoy laboran en el área de ciencias básicas de la carrera de Medicina dentro de la Facultad de Ciencias Médicas la mayor cantidad se encuentra en un rango de edad entre los 40 a los 59 años con un $82.4 \%$, el resto se encuentra entre 20 a 39 un $12.2 \%$ y entre 60 a 80 un $5.4 \%$

Tabla 2. Distribución de los docentes atendiendo a la edad

\begin{tabular}{clc} 
Grupos de & & Cantidad \\
\cline { 2 - 3 } edades & No. & $\%$ \\
\hline De 20 a 39 & 9 & 12.2 \\
De 40 a 59 & 61 & 82.4 \\
De 60 a 80 & 4 & 5.4 \\
Total & 74 & 100 \\
\hline
\end{tabular}

Fuente: Elaboración propia

En la tabla 3 se muestra los niveles de actitud que declaran tener estos profesores ante la importancia que le atribuyen a la utilización de las tecnologías de la información y las comunicaciones en el proceso enseñanza- aprendizaje, donde más del $50 \%$ es decir el $58.1 \%$ de los profesores de la Facultad de Ciencias Médicas pertenecientes al área básica de la carrera de medicina declaran tener una actitud excelente ante el uso de las tecnologías en la docencia, el $35.1 \%$ son aceptables y el $6.8 \%$ declaran una mala actitud. La tabla nos muestra en sentido creciente que los profesores están a favor del uso de las tecnologías con más del $50 \%$ en todos los ítems.

Tabla 3. Actitud ante las tecnologías de la información

\begin{tabular}{lccccc}
\hline \multicolumn{5}{c}{ Niveles de la actitud } \\
\hline Mo. & $\%$ & No. & $\%$ & Aceptable & Excelente \\
\hline 5 & 6.8 & 26 & 35.1 & 43 & $\%$ \\
\hline
\end{tabular}

Fuente: Elaboración propia

En la tabla 4 se muestra la relación entre actitud ante el uso de las tecnologías y la edad de los profesores donde se observa que los profesores que tienen una edad relativa entre 20 a 39 años son los usan más las tecnologías pues tienen un uso medio de $6.8 \%$ y uno alto de $5.4 \%$ por lo que todos la usan y los de 60 a 80 son los que tienen un uso más bajo con $2.7 \%$ y medio de $2.7 \%$ y no hacen uso alto de ellas 
Tabla 4. Actitud ante el uso de las tecnologías atendiendo a la edad

\begin{tabular}{|c|c|c|c|c|c|c|c|c|}
\hline \multirow{3}{*}{ Edades } & \multicolumn{6}{|c|}{ Uso de las TIC } & \multirow{2}{*}{\multicolumn{2}{|c|}{ Total }} \\
\hline & \multicolumn{2}{|c|}{ Bajo } & \multicolumn{2}{|c|}{ Medio } & \multicolumn{2}{|c|}{ Alto } & & \\
\hline & No. & $\%$ & No. & $\%$ & No. & $\%$ & No. & $\%$ \\
\hline De 20 a 39 & 0 & 0 & 5 & 6.8 & 4 & 5.4 & 9 & 12.2 \\
\hline De 40 a 59 & 5 & 6.8 & 14 & 18.9 & 42 & 56.7 & 61 & 82.4 \\
\hline De 60 a 80 & 2 & 2.7 & 2 & 2.7 & 0 & 0 & 4 & 5.4 \\
\hline Total & 7 & 9.5 & 21 & 28.4 & 44 & 62.1 & 74 & 100 \\
\hline
\end{tabular}

Fuente: Elaboración propia

Cuba se encuentra en un proceso de perfeccionamiento de su modelo económico en todos los sectores, en el que se reconoce el papel de la informatización de la sociedad como motor impulsor para su propio desarrollo (Vialart, 2018) (PCC,2016) (García, 2017). En este contexto, la Dirección Nacional de Informática y Comunicaciones del MINSAP trabaja en la actualización de la base legal y el plan de desarrollo y uso de las tecnologías de la información y las comunicaciones hasta el 2030. (Catelli, 2017) (MINSAP, 2017)

En correspondencia con el MINSAP y con esta era digital, donde la tecnología ocupa un papel preponderante dentro los procesos de enseñanza- aprendizaje en las aulas universitarias, se hace necesario que los profesores, que no son nativos digitales, adopten actitudes a favor de un pensamiento transformador que les permita desarrollar habilidades para poder lidiar mejor con las tecnologías de la información y las comunicaciones en función de una didáctica desarrolladora, dándole utilidad a la zona de desarrollo próximo declarado en el enfoque histórico cultural de Vigotsky, y cumpliendo con el rol de ser buenos guías en el aprendizaje de los estudiantes, especialmente mediado por tecnologías.(Zelada, 2018)

Las tecnologías de la información y las comunicaciones llegaron para acompañar los espacios que ocupan las libretas, los libros impresos, las tizas y el pizarrón, pues se suman al grupo de herramientas de que hoy se dispone para aprender, no son ni mejores ni peores, son otras que ofrecen muchas ventajas sin sustituirlas, para lograr modificar los ambientes educativos que existen, la responsabilidad está en cómo es capaz el profesor universitario de transformarse a la par de sus estudiantes y avanzar hacia el mundo tecnológico que se avecina cada vez con más fuerza.(Vela,2015)(Horrutinier, 2007)

Lo favorable del estudio realizado se muestra en sentido creciente que los profesores declaran una actitud a favor del uso de las tecnologías de la información y las 
comunicaciones para fomentar la implicación en los procesos de enseñanza y aprendizaje, otros declaran considerarlo imprescindible. Aunque existe todavía los que mantienen una actitud indiferente ante el uso de las mismas y niegan lo novedoso de su utilización.

En correspondencia los resultados mostraron que en el área básica de la Universidad de Ciencias Médicas de Matanzas laboran 74 profesores que imparten docencia a la carrera de medicina, en su mayoría mujeres con un rango de edad entre los 40 y 59 años. Todos con un alto nivel profesional y entrega, que están a favor de transformarse y avanzar en el proceso

Lo limitado del estudio es que se utilizó como muestra a los profesores de la carrera de medicina pertenecientes al área básica, pero se pudiera hacer extensivo no solo al área clínica sino a todas las carreras de las Ciencias Médicas dentro de la Universidad tanto del pregrado como del postgrado lo que permite obtener una información más completa sobre el problema a resolver dentro de la Universidad y realizar acciones para potencializar el uso que se realiza de estas tecnologías.

Los autores de esta investigación así como Cardona (2016), Rivero (2014) y Cabrales (2017) coinciden con Rodríguez (2016) cuando plantea que :"En el contexto, de una sociedad que avanza en la informatización; de un aumento en los niveles de acceso y las formas de conexión tecnológica, sobre todo entre los más jóvenes y de proyecciones que apuntan al incremento de las opciones de conectividad a Internet en la sociedad, la educación tiene el imperativo de convertirse en la «punta de lanza» en materia de formar conductas responsables ante el uso de las tecnologías de la información y las comunicaciones, y de generar capacidades para aplicarlas de forma coherente en el proceso de enseñanza aprendizaje".

En consecuencia este proceso debe convertirse en un quehacer tecnocientífico, donde ciencia en su carácter investigativo y tecnología se imbrican en un proceso cognoscitivo, donde sujeto y objeto de la educación constituyen elementos conscientes y activos, el docente es un moderador que orienta, controla y evalúa la actividad de estudio, la capacidad de aplicación de la realidad estudiada y el desarrollo de capacidades, habilidades y destrezas en la aplicación de los conocimientos sino se prepara para ello le será difícil incorporar a la sociedad un profesional más capaz con posibilidades de utilizar la tecnología moderna y los procesos tecnocientífico de punta sustentados en un conocimiento verdadero.(Alfonso, 2015)

\section{Conclusiones.}

- Sin duda las tecnologías pueden suministrar medios para la mejora de los procesos de enseñanza aprendizaje, pero su utilización a favor o en contra de una sociedad 
más desarrollada, dependerá en gran medida de la educación, de los conocimientos y la capacidad crítica de sus usuarios, que son las personas que ahora estamos formando.

- El mundo nuevo requiere la escuela nueva, dijo José Martí. Ganar la batalla política-cultural que se libra hoy en el terreno de la comunicación, pasa por formar un educando más consciente y responsable sobre el papel de las tecnologías de la información y las comunicaciones.

- La educación es la clave para lograrlo y el profesor su guía principal, por lo que consideran los autores no solo es necesaria la actitud, sino que debe existir la aptitud utilizar las tecnologías a favor del proceso enseñanza aprendizaje en el pregrado y fortalecer el desarrollo y la creatividad del proceso hasta lograr un profesional más preparado e independiente.

\section{Referencias bibliográficas.}

Alfonso GY, Sánchez GS, Guerrero GA, et al (2015). Software educativo en el proceso de enseñanza-aprendizaje de la asignatura Ética y Bioética. Rev Ciencias Médicas.; 19(1):89-99. https://www.medigraphic.com/cgi-bin/new/resumen.cgi?idarticulo=56079

Álvarez-Espinoza, A; Sebastián Balmaceda, Ch (2018) El concepto dialéctico de internalización en Vygotski: aproximaciones a un debate Psicología, Conocimiento y Sociedad 8(1), 5-35 Trabajos originales ISSN: 1688-7026 https://revista.psico.edu.uy/index.php/revpsicologia/article/view/493

Cabrales. O, Díaz. V. (2017) El aprendizaje autónomo en los nativos digitales. Conhecimento \& Diversidade, Niterói, v. 9, n. 17, p. 12-32, jan. /jun. https://revistas.unilasalle.edu.br/index.php/conhecimento_diversidade/article/view/3 $\underline{473}$

Cardona, S., Vélez, J., \& Tobón, S. (2016) Contribución de la evaluación socio formativa al rendimiento académico en pregrado. Educar,. 52 (2), 423-447. Recuperado de: http://www.raco.cat/index.php/Educar/article/view/311816/401877

Catelli, Jr., R. (2017) Los exámenes nacionales de certificación para jóvenes y adultos en el contexto de las políticas públicas de educación en Brasil, Chile y México. Revista Interamericana de Educación de Adultos, 39(1), 49-68. https://www.crefal.org/rieda/images/rieda-2017-1/exploraciones2.pdf

Chancusig Chisag, JC., Flores Lagla, GA, Fernanda Constante, M. (2017) Las TICs En La Formación De Los Docentes Boletín virtual - vol 6 - 2 https://revista.redipe.org/index.php/1/article/view/206

García, C. (2017) Modelo de millennials emprendedor. Cambios \& Permanencias, 8 (2), $179-195$ https://revistas.uis.edu.co/index.php/revistacyp/article/view/7788 
Horruitiner Silva, P (2007) Introducción Del Libro La Universidad Cubana: El Modelo De Formación. Revista Pedagogía Universitaria Vol. XII No. 4

Ministerio de Salud Pública. (2017) Plan de desarrollo y uso de las Tecnologías de la Información y Comunicaciones del Sistema Nacional de Salud 2017-2021. Rev Infodir. ～25:133-57. Disponible en: http://revinfodir.sld.cu/index.php/infodir/article/view/432/512

Partido Comunista de Cuba (2016). Actualización de los Lineamientos de la Política Económica y Social del Partido y la Revolución para el Período 2016-2021. La Habana: Editora Política;. Disponible en: http://www.granma.cu/file/pdf/gaceta/01Folleto.Lineamientos-4. Pdf Acceso el 14 de abril de 2017

Rivero López, MS, et al. (2014) Elementos de una estrategia para el desarrollo de la Educación a Distancia en la Universidad de Ciencias Médicas de La Habana. Educación Médica Superior, vol. 28, no 2, p. 292-300. http://scielo.sld.cu/pdf/ems/v28n4/ems05414.pdf

Rodero C. et al. (2015) Análisis de la fiabilidad y validez de un cuestionario docente. A: JENUI. "Actas de las XXI Jornadas de la Enseñanza Universitaria de la Informática". Universitat Oberta La Salle ed. Andorra la Vella: Universitat Oberta La Salle, 2015, p. 136-143. http://hdl.handle.net/2117/76844

Rodríguez Guerrero, L., Martínez Molina, J., Merencio Cautín, JL., (2017) Educación «en línea» con las tecnologías Periódico Granma.

http://www.granma.cu/cuba/2017-06-22/educacion-en-linea-con-las-tecnologias22- 06-2017-22-06-02

Rodríguez Selpa, S et al (2016) Sistema de medios de enseñanza-aprendizaje para la disciplina Centro de Desarrollo de las Ciencias Sociales y Humanísticas en Salud (3):532-548

http://www.humanidadesmedicas.sld.cu/index.php/hm/article/view/1007

Vela Valdés, J (2015) Regulaciones e importancia del trabajo metodológico en la Educación Médica Superior. Escuela Nacional de Salud Pública. https://www.medigraphic.com/cgi-bin/new/resumen.cgi?IDARTICULO=64796

Vialart Vidal,MN (2017) Programa Educativo para el empleo de los Entornos Virtuales de Enseñanza Aprendizaje dirigido a los docentes de enfermería. Tesis de Doctorado http://www.revtecnologia.sld.cu/index.php/tec/article/view/1025

Vialart Vidal MN, Vidal Ledo MJ, Sarduy Domínguez Y, Delgado Ramos A, Rodríguez Díaz A, Fleitas Estévez I, (2018) Aplicación de la eSalud en el contexto cubano. Rev Panam Salud Pública. ;42: e19.

https://doi.org/10.26633/RPSP.2018.19. 
Zelada Pérez. M (2018) Modelo curricular para el desarrollo de competencias informacionales en los profesores de la Universidad de Ciencias Médicas de La Habana. Tesis de Doctorado http://tesis.sld.cu/index.php?P=DownloadFile\&Id=744

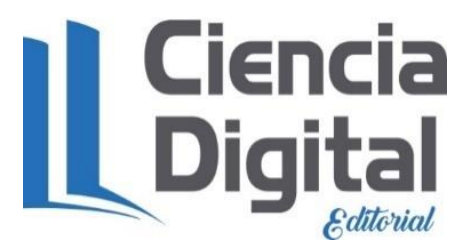


PARA CITAR EL ARTÍCULO INDEXADO.

Naranjo Rodríguez, S., Almanza Santana, L., Rodríguez Almanza, L., \& Delgado Almanza, R. (2021). Actitud de los profesores ante el uso de las TIC en la Facultad de Ciencias Médicas de Matanzas. Anatomía Digital, 4(1), 29-39. https://doi.org/10.33262/anatomiadigital.v4i1.1479

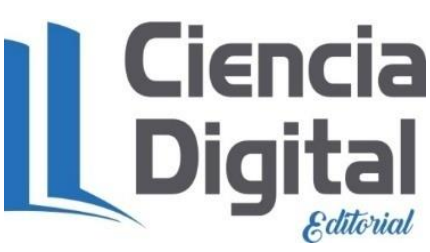

El artículo que se publica es de exclusiva responsabilidad de los autores y no necesariamente reflejan el pensamiento de la Revista Anatomía Digital.

El artículo queda en propiedad de la revista y, por tanto, su publicación parcial y/o total en otro medio tiene que ser autorizado por el director de la Revista Anatomía Digital.
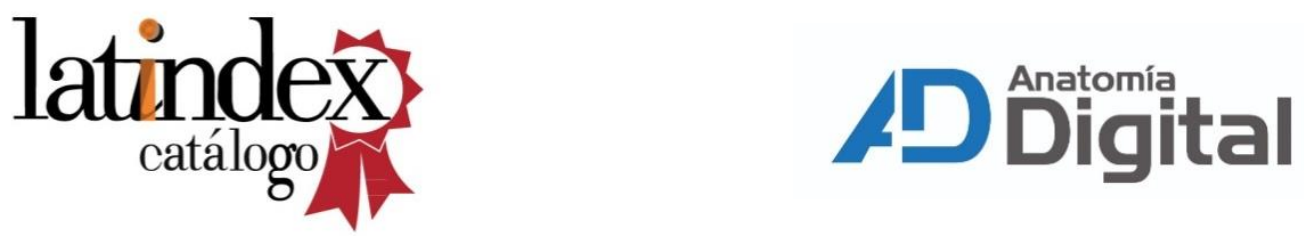